\title{
Primary murine neurons as in vitro model for studying neuroinfections caused by human adenoviruses
}

\author{
J. CYMERYS ${ }^{1 *}$, A. SŁOŃSKA ${ }^{1,2 *}$, M. CHODKOWSKI ${ }^{1}$, M. PRZYBYLSKI ${ }^{3}$, M. W. BAŃBURA ${ }^{1}$
}

\begin{abstract}
${ }^{1}$ Division of Microbiology, Department of Preclinical Sciences, Faculty of Veterinary Medicine, Warsaw University of Life Sciences - SGGW, Ciszewskiego 8, 02-786 Warsaw, Poland; ${ }^{2}$ Division of Physiology, Department of Physiological Sciences, Faculty of Veterinary Medicine, Warsaw University of Life Sciences - SGGW, Nowoursynowska 159, 02-776 Warsaw, Poland; ${ }^{3}$ Chair and Department of Medical Microbiology, Faculty of Medicine, Medical University of Warsaw, Poland
\end{abstract}

\begin{abstract}
Summary. - Adenoviral infections of the central nervous system are rare, but they are characteristic for their high mortality rate. People with impaired immunity and children are particularly vulnerable. A few reports of neuroinfections caused by adenoviruses are found in literature. In this study the tropism of the human adenoviruses type 4, 5, 7 to primary cultures of murine neurons and the influence of infection on the neuron morphology and actin cytoskeleton was examined. The A549 non-small-cell lung cancer cell line was used as a reference line. Viral effects upon the cell culture were evaluated by direct immunofluorescence. The levels of adenovirus replication in the above-mentioned cell cultures were determined by real-time PCR. In the current study we demonstrated for the first time that human adenovirus (HAdV) type 4, 5 and 7 exhibits tropism for neurons cultured in vitro followed by the extensive replication of all serotypes in the primary culture of murine neurons. Immunofluorescent labelling and confocal microscopy revealed the changes in cell morphology, destruction of actin cytoskeleton and cell lysis as the final stage of infection. According to the obtained results we can assume that productive cycle of HAdV in the studied cell cultures occurred. We also observed accumulation of nuclear actin within nuclei of infected cells which may indicate that it plays role in adenovirus infection and replication in neurons and A549 cells.
\end{abstract}

Keywords: adenovirus; neuroinfection; primary culture of murine neurons; actin cytoskeleton; in vitro model

\section{Introduction}

Adenoviruses are ubiquitous in human and animal populations worldwide, capable of infecting upper respiratory tract, digestive tract and eyes, but due to the different tissue tropism, adenoviruses can also cause infections of other organ systems (Hayashi and Hogg, 2007). Moreover, data available in literature indicate that human adenoviruses

E-mail: jcymerys@op.pl; phone: +4822-593-60-60. "Both authors equally contributed to the paper.

Abbreviations: $\mathrm{CAR}=$ coxsackievirus-adenovirus receptor; $\mathrm{CPE}=$ cytopathic effect; $\mathrm{HAdV}(\mathrm{s})$ = human adenovirus(es); p.i. $=$ post infection
(HAdVs) can be an etiological factor of infections in the central nervous system. Although neurological complications of adenoviral infection are rare, they are usually morbid with the high risk of mortality. Chatterjee et al. (2000) reported the isolation of HAdV 5 from the brain of an infant who developed skin rash, pulmonary congestion, and fever and who died 6 days later.

Mechanisms of adenoviral neuroinfections are poorly known and, what is worth noting, there are no data concerning the use of primary neuronal culture for studying adenoviral infection in vitro. Replication of HAdV in murine neuronal cells is possible due to the presence of coxsackievirus-adenovirus receptor (CAR), necessary for an initial stage of adenoviral infection. Expression of CAR receptor in the central and peripheral nervous system of murine 
fetus is abundant, while very weak in adult mice (Dorner et al., 2005). The strongest CAR expression is detected on the ependymal cells of cerebral ventricles. In humans, very

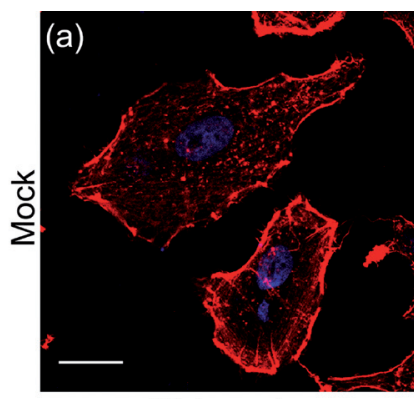

24 hrs p.i.
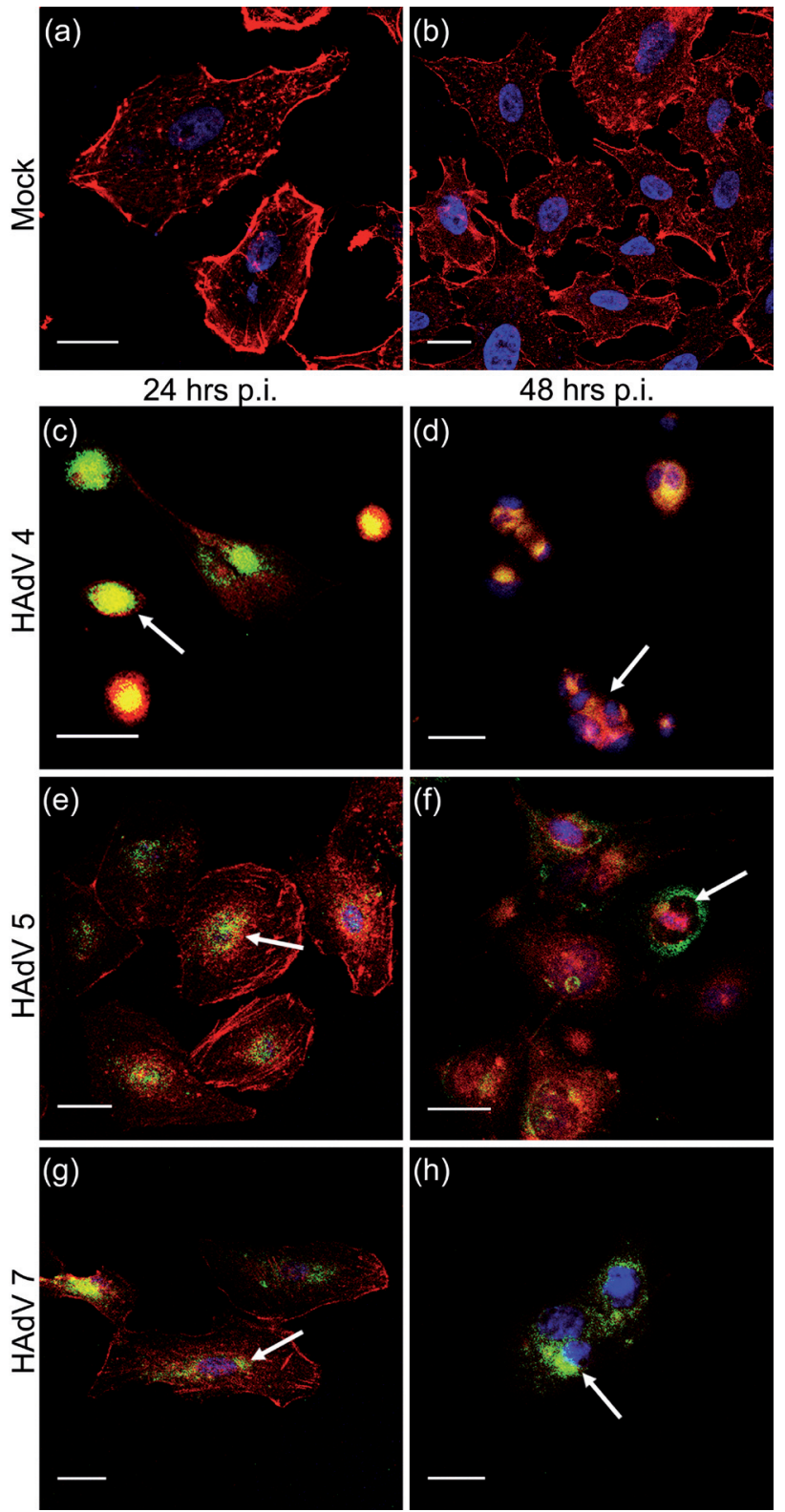

$48 \mathrm{hrs}$ p.i.
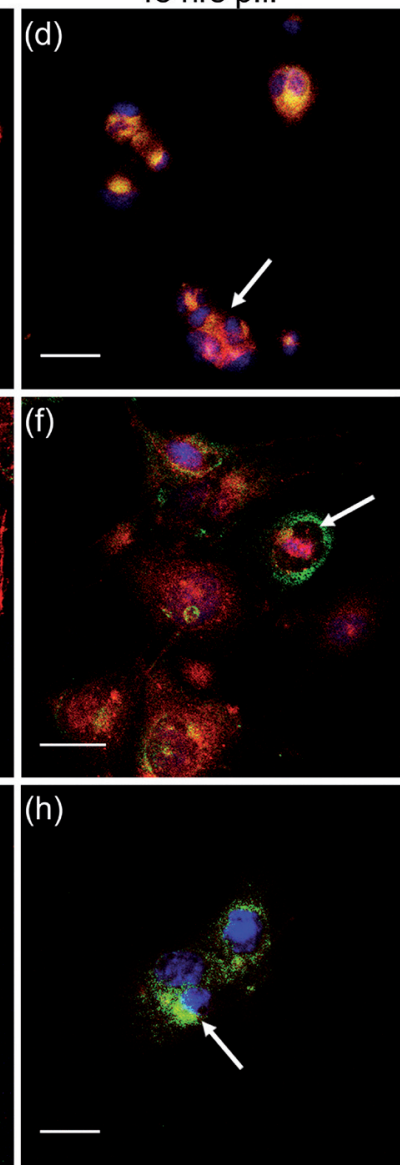

Fig. 1

The effect of HAdV infection on the cell morphology and structure of the actin cytoskeleton in A549 cells

Confocal images of mock-treated (a, b), HAdV 4 infected (c, d) HAdV 5 infected (e,f) and HAdV 7 infected (g, h) A549 cells. Actin filaments are shown in red, EHV-1 antigens in green and DNA in blue. In mock infected cells, microfilaments formed a network of fibres within the cytoplasm, however, the densest distribution was detected in the cortical layer of the cytoplasm in the peripheral region of the plasma membrane $(a, b)$. In HAdV infected cells (24 and $48 \mathrm{hr}$ p.i.) microfilaments were disrupted. CPE manifested by disintegration and nucleus degradation, which led to cell degeneration. Arrows indicate viral antigen inside cells. Scale bars $-20 \mu \mathrm{m}$. strong expression of CAR is also found in some tumors of the central nervous system, such as medulloblastoma and neuroma. It is therefore possible to treat these tumors with gene therapy based on adenovirus vectors (Yuonming and Jeffrey, 2005).

Therefore, in the current study we have investigated the replication kinetics of HAdV serotype 4, 5 and 7 in primary cultures of murine neurons and A549 cell line, which was used as a positive control. Additionally, we have also examined the changes in the actin network of primary murine neurons and A549 cells induced by different HAdV serotypes.

\section{Materials and Methods}

Cells and adenoviruses. Balb/c ( $\left.\mathrm{H}-2^{\mathrm{d}}\right)$ mice genetically susceptible to EHV-1 infection were used to establish primary culture of murine neurons, as described before (Cymerys et al., 2010). Primary murine neurons were cultured in B-27 Neuron plating medium, consisting of neurobasal medium (Gibco Life Technologies), B-27 supplement (Gibco Life Technologies), 200 mmol/l glutamine, 10 $\mathrm{mmol} / \mathrm{l}$ glutamate and penicillin/streptomycin antibiotics with $10 \%$ supplemental fetal bovine and equine serum (Gibco Life Technologies). Cells were plated onto poly-D-lysine with laminin - coated sterile coverslips at a density of $5 \times 10^{4}$ neurons per well $\left(3.6 \mathrm{~cm}^{2}\right)$ and maintained at $37^{\circ} \mathrm{C}$ in $5 \% \mathrm{CO}_{2}$. Four days after plating, the medium was removed and replaced with Neuron feeding medium (B-27 Neuron plating medium without glutamate). Murine neurons were maintained in this medium for next 6 days prior to treatments. A549 cell line (non-small-cell lung cancer) was cultured in Modified Eagle's medium (MEM), with $2 \mathrm{mmol} / \mathrm{l} \mathrm{L}$-glutamine, $10 \% \mathrm{FBS} / \mathrm{FCS}$ and antibiotics $-10 \mathrm{U} / \mathrm{ml}$ penicillin and $100 \mu \mathrm{g} / \mathrm{ml}$ streptomycin. Cell cultures were incubated at $37^{\circ} \mathrm{C}$ with $5 \% \mathrm{CO}_{2}$. Primary murine neuron cultures and A549 cell line were infected with human adenovirus serotype 4 (HAdV 4 species E, RI-67, ATCC VR-1572), serotype 5 (HAdV 5 species C, Adenoid 75, ATCC VR-5) or serotype 7 (HAdV 7 species B, Gomen, ATCC VR-7) at $10^{5} \mathrm{CCID}_{50} / \mathrm{ml}$ in culture medium. After incubation at $37^{\circ} \mathrm{C}$ for $1 \mathrm{hr}$, the inoculum was aspirated and fresh culture medium was added. Subsequently, infected cells were incubated for 24 or $48 \mathrm{hr}$ at $37^{\circ} \mathrm{C}$ with $5 \% \mathrm{CO}_{2}$.

Immunofluorescent staining and confocal microscopy. For immunofluorescent staining, cells were plated onto laminin-coated coverslips. After 24 and $48 \mathrm{hr}$ post infection (p.i.), cells were fixed in $3.7 \%$ paraformaldehyde/PBS (Sigma Aldrich) for $30 \mathrm{~min}$ at room temperature, permeabilized in $0.5 \%$ Tween/PBS for $5 \mathrm{~min}$, washed in PBS and blocked with PBS containing $1 \%$ bovine serum albumin (BSA) (Sigma Aldrich). Filament structures of actin were visualized using TRITC-phalloidin conjugate (500 ng/ml; Sigma Aldrich). Polyclonal antiserum ADENO MAB conjugated to FITC (Light Diagnostics ${ }^{\text {Tx }}$, Merck Millipore) was used to detect viral antigens. Cell nuclei were stained with bisbenzimidine/Hoechst 33258 ac- 
cording to the manufacturers' recommendations. Confocal images were captured using a FluoView FV500 laser scanning confocal microscope and analyzed with FluoView 3.1. software (Olympus, Poland), ImageJ, and Adobe Photoshop software.

Real-time PCR. The quantity of the HAdV DNA in all tested samples was estimated using real-time PCR (qPCR) technique described by Rola et al. (2007), with further modifications (Rynans et al., 2015). Each amplification reaction embraced, except tested samples, also positive HAdV calibrators in range 100-10,000,000 copies/ml and negative control of DNA extraction and amplification process. All qPCR tests were run on LightCycler 480 instrument, using LightCycler TaqMan Master amplification kit (Roche Diagnostics). Fluorescence levels were measured at $560 \mathrm{~nm}$ wavelength, typical for JOE fluorescent dye, and a threshold cycle $(C t)$ value for each sample was calculated. $C t$ values of HAdV calibrators were the basis for standard curves and the copy numbers were calculated automatically by a software package for data analysis.

Statistical analysis. Results of three independent experiments were statistically evaluated by one-way analysis of variation (ANOVA) using the Student-Newman-Keuls multiple comparisons test. This analysis was performed using GraphPad PrismTM version 4.03 software (GraphPad Software Inc., USA). Statistical differences were interpreted as significant at $P<0.05$, highly significant at $P<0.01$ and non-significant at $P>0.05$.

\section{Results and Discussion}

In the present study, we demonstrated that all used serotypes of human adenoviruses - HAdV 4, 5, and 7 were able to infect primary murine neurons and A549 cell lines, without the need for initial adaptation. Infected cells exhibited morphological changes, followed by cell lysis at the final stage of infection. These observations indicated that productive replication cycle of HAdV in the both tested cell cultures has occurred. In primary murine neurons infected with HAdV 4, 5 or 7 , cytopathic effect (CPE) was well visible as early as 24 hr p.i. and manifested by disintegration and nucleus degradation, changes in a cell shape and rearrangements of the actin filaments, which led to cell degeneration. Moreover, in cells which did not undergo lysis, accumulation of viral antigens in the cytoplasm, accompanied by increased density of actin fibres was detected (Fig. 1, arrows). Infection of A549 cell line led to a significant decrease in the number of cells. The $\mathrm{CPE}$ occurred quite early (before $24 \mathrm{hr}$ p.i.) in comparison to primary cultures of murine neurons and the following changes were observed: disintegration and degradation of a nucleus, rearrangements of the actin filaments, changes of cell shape and finally destruction of cell. As for neurons, accumulation of viral antigens around the nucleus was apparent (Fig. 2, arrows). Dramatic reorganization of the cell nucleus during intermediate and late phase of productive adenovirus infection was previously reported but mainly in HeLa cell line (Pombo et al., 1994; Gama-Carvalho et al., 2003). HAdVs induced formation of ring and crescent-

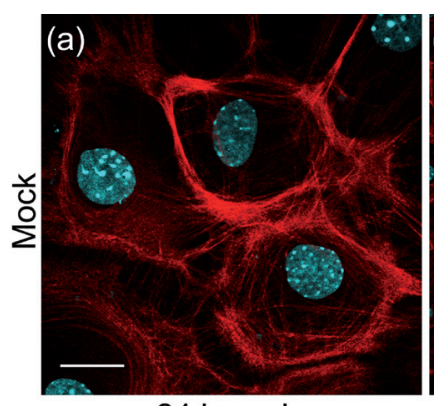

24 hrs p.i.
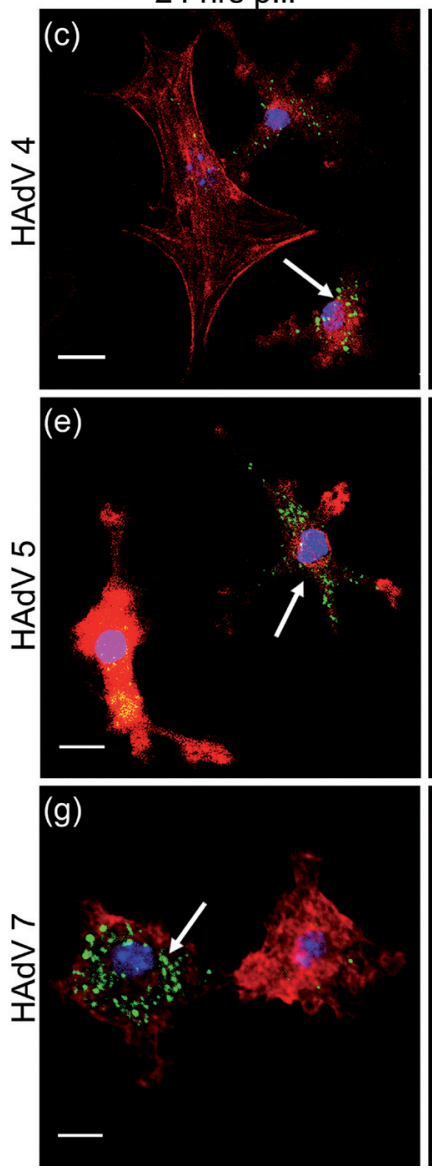

Fig. 2

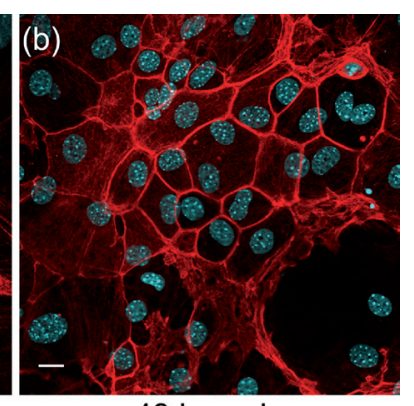

48 hrs p.i.
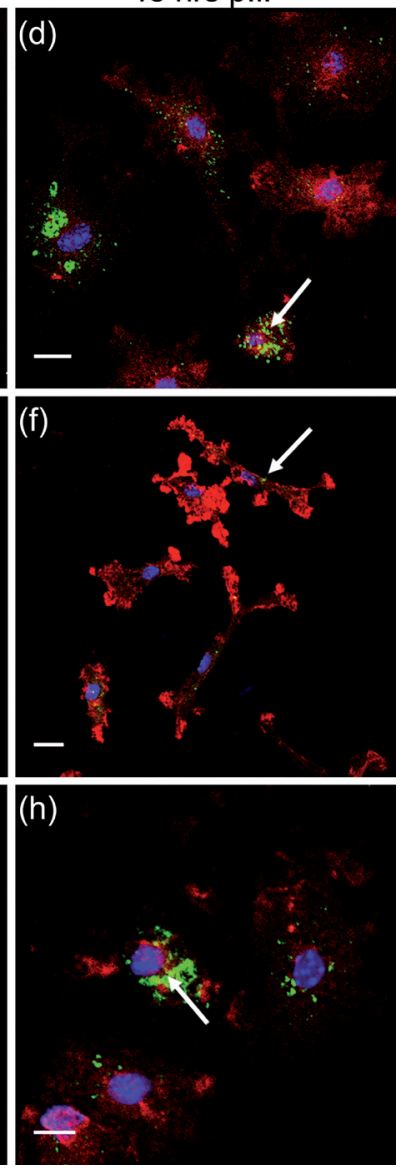

The effect of HAdV infection on the cell morphology and structure of the actin cytoskeleton in primary murine neurons

Confocal images of mock-treated (a, b), HAdV 4 infected (c, d) HAdV 5 infected (e, f) and HAdV 7 infected (g, h) primary murine neurons. Actin filaments are shown in red, EHV-1 antigens in green and DNA in blue. In mock infected cells, microfilaments formed a network of fibres within the cytoplasm, however, the densest distribution was detected in the cortical layer of the cytoplasm in the peripheral region of the plasma membrane (a, b). In HAdV infected cells ( 24 and $48 \mathrm{hr}$ p.i.) microfilaments were disrupted. CPE manifested by disintegration and degradation of a nucleus, changes of cell shape and finally destruction of cell. Arrows indicate viral antigen inside cells. Scale bars $-20 \mu \mathrm{m}$. 
like structures, which demarcated area of active viral DNA replication. Moreover, Fuchsova et al. (2015) reported that nuclear actin was recruited to viral centers during the late phase of infection and therefore they concluded that it played important role in adenovirus infection and replication. In our studies, we also detected the accumulation of nuclear actin within the nucleus of infected primary murine neurons (Fig. 3c), as well as A549 cells (Fig. 3a) for all tested HAdV serotypes. Co-localization of nuclear actin and viral antigen was confirmed by the measurement of the fluorescence intensity, in which the signals corresponding to actin and HAdV antigen overlapped (Fig. 3b,d). According to these observations we can assume that nuclear actin may also be involved in viral replication in primary murine neurons and A549 cells.

The quantitative PCR analysis showed a statistically significant increase in the amount of viral DNA of all analyzed HAdV serotypes, both in murine neurons and A549 cells, in comparison to the uninfected control (Fig. 4). The highest, statistically significant increase in the amount of viral DNA

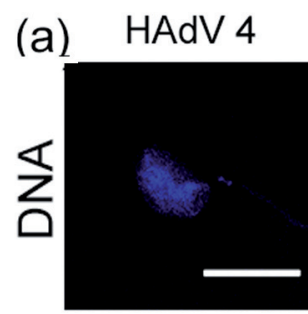

HAdV 5
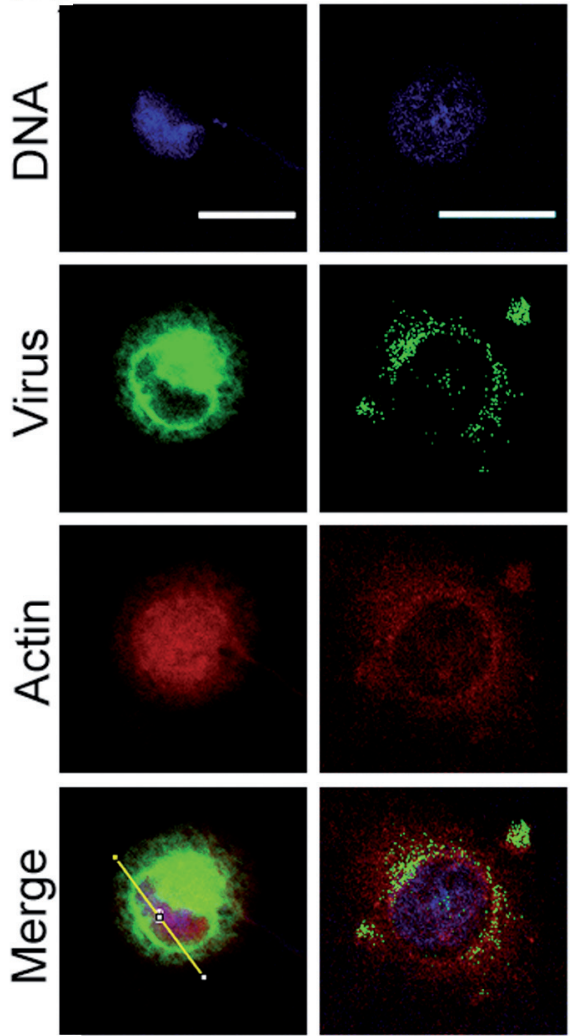

(b)

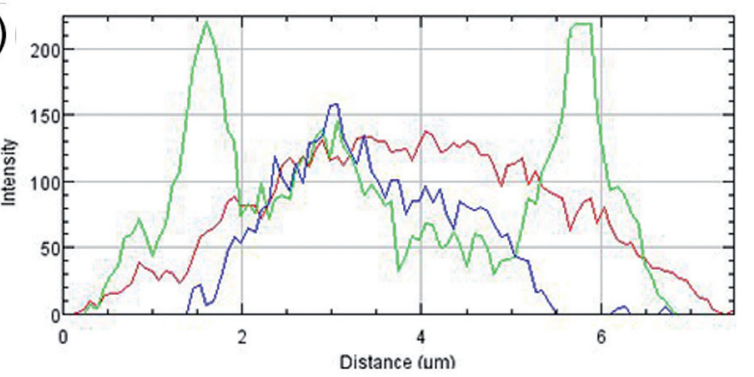

HAdV 7
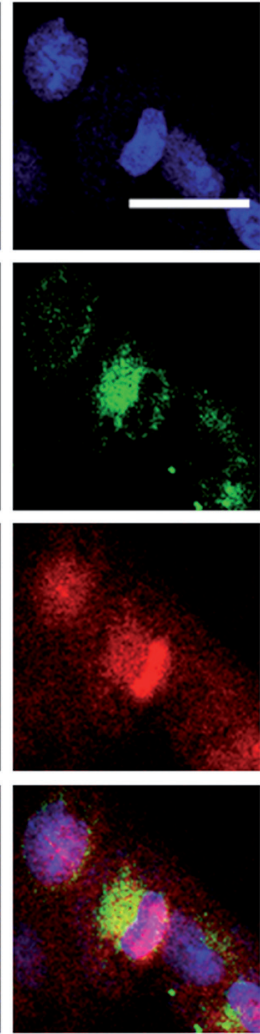

(d)

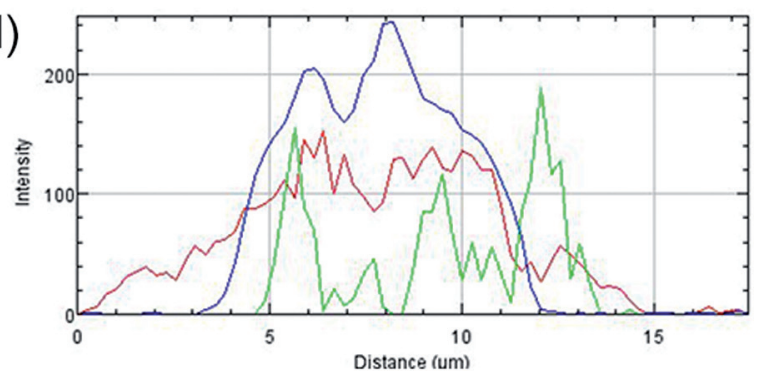

Fig. 3

Accumulation of nuclear actin within nuclei of HAdV infected A549 cells and neurons

Confocal images of HAdV 4, 5 or 7 infected A549 cells (a) and primary murine neurons (c). Actin filaments are shown in red, EHV-1 antigens in green and DNA in blue. Profile plot of fluorescence signal intensities along the yellow lines indicate the co-localisation of actin and viral antigen within the nucleus of infected cells (b, d). Scale bars - $10 \mu \mathrm{m}$. 

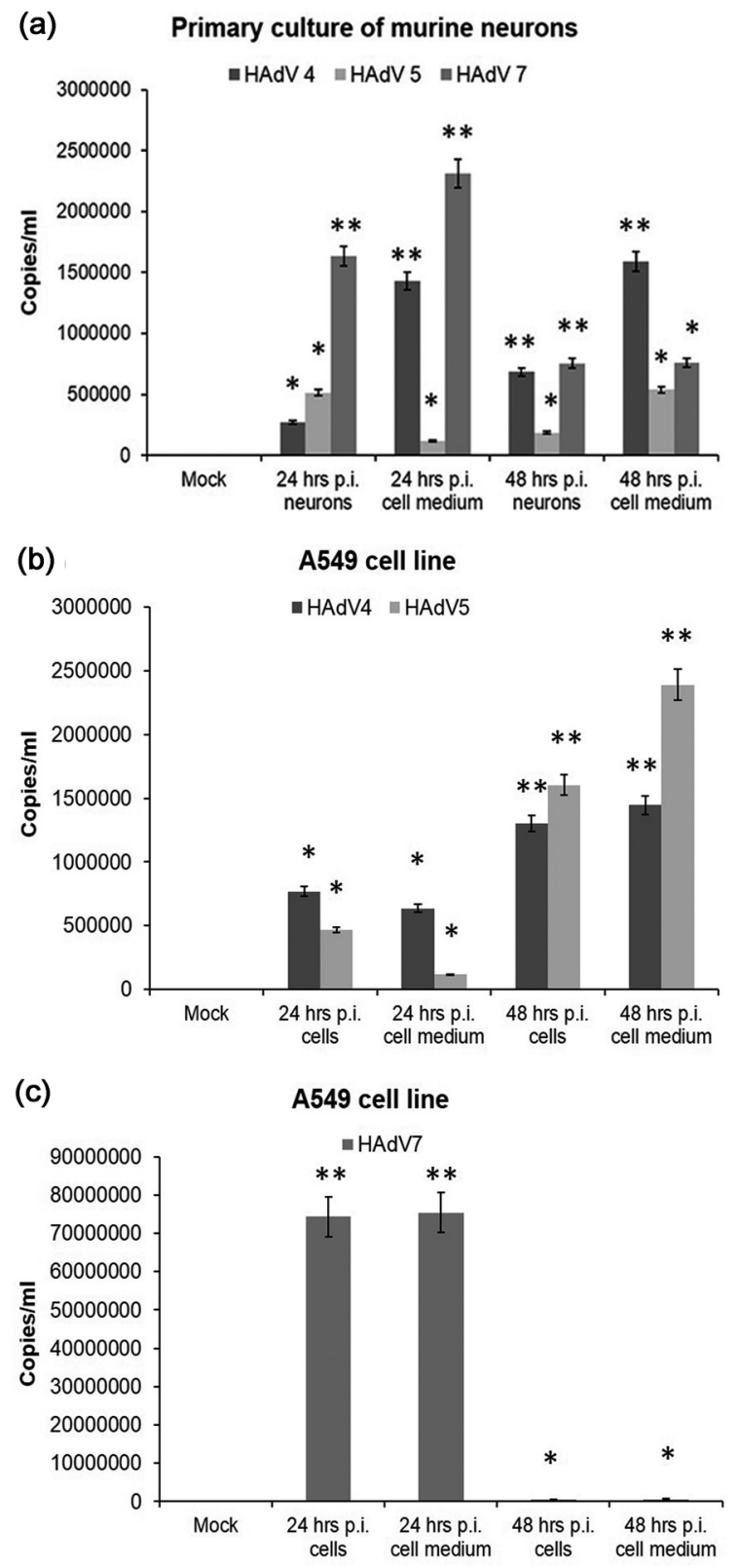

Fig. 4

Viral DNA levels in primary murine neurons and A549 cell line infected with various strains of HAdV

Comparison of viral DNA level in mock-infected cells, neurons and A549 cells infected with analyzed HAdV serotypes ( $24 \mathrm{hr}$ p.i. and $48 \mathrm{hr}$ p.i.) by qPCR $\left({ }^{*}, P<0.01{ }^{*}, P<0.05\right)$.

was observed in A549 cells, which is consistent with the literature data (Fig. 4b,c). Replication in neurons was less efficient (Fig. 4a). It should be noted that A549 cell line is commonly used for multiplication of different HAdV serotypes in vitro, both in viral diagnostics and research (Dzieciątkowski et al., 2008). Moreover, A549 cell line is a golden standard in replication studies of human adenoviruses in vitro. In this study, HAdV 7 reached the highest replication level in A549 cells at $24 \mathrm{hr}$ p.i. $\left(7.01 \pm 2.19 \times 10^{7}\right.$ copies $\left./ \mathrm{ml} ; P<0.01\right)$, while HAdV 5 showed the lowest level of viral DNA $(4.6 \pm 1.25 \mathrm{x}$ $10^{5}$ copies $/ \mathrm{ml} ; P<0.05$ ), but it was also statistically significant increase in comparison to uninfected control (Fig. 4b,c). Interestingly, similar results were obtained for primary cultures of murine neurons. The highest, statistically significant increase in the amount of viral DNA was observed for HAdV 7 at 24 hr p.i. $\left(1.6 \pm 1.01 \times 10^{6}\right.$ copies $\left./ \mathrm{ml} ; P<0.01\right)$, and lower statistically significant increase for HAdV $5\left(5.1 \pm 1.65 \times 10^{5}\right.$ copies/ml; $P<0.05)$ and HAdV $4\left(2.7 \pm 0.94 \times 10^{5}\right.$ copies $/ \mathrm{ml}$; $P<0.05$ ), in comparison to uninfected control (Fig. $4 \mathrm{a})$. The differences observed for viral titers in neurons depend on the HAdV serotype and are probably related with the different tropism of adenoviruses for particular cell types. According to the literature data, serotypes used in this study show tropism mostly for the cells of the respiratory tract causing mild infection. Additionally, HAdV 7 could be isolated from the cells of nervous and urinary systems.

As already mentioned above, little information is available on the replication of HAdVs in neuronal cells. Serotypes 2, $7,5,11,19,31,32$, and 49 were isolated from the central nervous system (Chatterjee et al., 2000). Their presence was related with diseases such as: meningitis and encephalitis, myelitis, chronic persistent inflammation of the central nervous system and Rey-like syndrome (Rynans et al., 2013).

In summary, the results presented in this study are innovative, because we have proven for the first time the replication of HAdV 4 in primary murine neurons. So far, there were no reports on isolation of this serotype from the central nervous system. Obtained results provide a basis for testing other serotypes of HAdV in primary culture of murine neurons in vitro. Additionally, tropism of the above serotypes for nerve cells makes it possible to use them as vectors in treatment of diseases such as brain tumors or neurodegenerative diseases.

Acknowledgements. This work was supported by the grant No. NN308 028739 from Polish Ministry of Science and Higher Education. We would like to thank Dr. Tomasz Dzieciątkowski for critical review of the manuscript.

\section{References}

Chatterjee NK, Samsonoff WA, Balasubramaniam N, Rush-Wilson K, Spargo W, Church T M (2000): Isolation and characterization of adenovirus 5 from the brain of an infant with fatal cerebral edema. Clin. Infect. Dis. 31, 830-833. https:/doi.org/10.1086/314041

Cymerys J, Dzieciątkowski T, Słońska A, Bierła J, Tucholska A, Chmielewska A, Golke A Bańbura MW (2010): Equine 
herpesvirus type 1 (EHV-1) replication in primary murine neurons culture. Pol. J. Vet. Sci. 13, 701-708. https:/doi. org/10.2478/v10181-010-0022-3

Dorner AA, Wegmann F, Butz S, Wolburg-Buchholz K, Wolburg H, Mack A, Vestweber D (2005): Coxsackievirus-adenovirus receptor (CAR) is essential for early embryonic cardiac development. J. Cell Sci. 118, 3509-3521. https:/doi. org $/ 10.1242 /$ jcs. 02476

Dzieciątkowski T, Rola A, Midak-Siewierska A (2008): Adenoviral infections in humans. Post. Mikrobiol. 47, 15-22.

Fuchsova B, Serebryannyy LA, de Lanerolle P (2015): Nuclear actin and myosins in adenovirus infection. Exp. Cell Res. 338, 170-182. https:/doi.org/10.1016/j.yexcr.2015.07.025

Gama-Carvalho M, Condado I, Carmo-Fonseca M (2003): Regulation of adenovirus alternative RNA splicing correlates with a reorganization of splicing factors in the nucleus. Exp. Cell Res. 289, 77-85. https:/doi.org/10.1016/S00144827(03)00251-9

Hayashi S, Hogg JC (2007): Adenovirus infections and lung disease. Curr. Opin. Pharmacol. 7, 237-243. https:/doi. org/10.1016/j.coph.2006.11.014
Pombo A, Ferreira J, Bridge E, Carmo-Fonseca M (1994): Adenovirus replication and transcription sites are spatially separated in the nucleus of infected cells. EMBO J. 13, 5075-5085.

Rola A, Przybylski M, Turowska A, Dzieciątkowski T (2007): Detection of human adenoviruses with real-time PCR assay using TaqMan fluorescent probes. Med. Dośw. Mikrobiol. 59, 371-377.

Rynans S, Dzieciątkowski T, Młynarczyk G (2013): Adenovirus infection in immunocompromised patients. Post. Hig. Med. Dośw. 67, 964-972.

Rynans S, Dzieciątkowski T, Przybylski M, Basak GW, Rusicka P, Tomaszewska A, Hałaburda K, Jędrzejczak WW, Młynarczyk G (2015): Incidence of adenoviral DNAemia in Polish adults undergoing allogeneic haematopoietic stem cell transplantation. Arch. Immunol. Ther. Exp. (Warsz) 63, 79-84. https:/doi.org/10.1007/s00005-014 -0320-Z

Yuonming Z, Jeffrey MB (2005): Adenovirus receptor. J. Virol. 79, 12125-12131. https:/doi.org/10.1128/JVI.79.19.12125$\underline{12131.2005}$ 